\title{
Phonophoresis in Adhesive Capsulitis (Frozen Shoulder)
}

\section{Sunam Kumar Barua ${ }^{1 *}$ \\ Zahangir Alam ${ }^{2}$}

${ }^{1}$ Department of Physical Medicine

Dhaka Medical College

Dhaka, Bangladesh.

${ }^{2}$ Department of Physical Medicine \& Rehabilitation Chattagram Maa- O- Shishu Hospital Medical College Chittagong, Bangladesh.
*Correspondence to:

Dr. Sunam Kumar Barua

Assistant Professor

Department of Physical Medicine

Dhaka Medical College

Dhaka, Bangladesh.

Mobile: +8801819190812

E-mail: sunambarua@gmail.com

\section{INTRODUCTION}

Adhesive capsulitis is a condition characterized by limitation of motion of the shoulder joint with pain at the extremes of motion. It was first described by Putaman in1882 and later by Codman. The initial presentation is pain, which is generalized and referred to the upper arm, back, and neck. As the pain increases, loss of joint motion ensues. The process is generally self limiting and in most cases resolves spontaneously unless there is an underlying problem. ${ }^{1}$ In the early phase, there is marked anterior joint/ capsular tenderness and stress pain in a capsular pattern; later there is painless restriction, often of all movements ${ }^{2}$.

Adhesive capsulitis - also known as frozen shoulder, pericapsulitis, scapulohumeral periarthritis or check- rein syndrome ${ }^{3}$. The condition usually resolves spontaneously after about 18 months $^{4}$. Adhesive capsulitis is a common musculoskeletal disorder mainly affecting middle aged adults ${ }^{5}$. Also self care activities and occupational activities decreases depends on which shoulder is involved, for instance, self care, grooming, combing hair, eating and dressing are impaired if right shoulder is involved for right handed person, on the other hand dressing and perineal care are hampered if left shoulder is involved.

Adhesive capsulitis should be divided into primary and secondary types. Subdivision of secondary adhesive capsulitis in to intrinsic, extrinsic and systemic types ${ }^{6}$. Primary adhesive capsulitis and idiopathic adhesive capsulitis are considered identical and not associated with a systemic condition or history of injury. Another classification system based on patient's irritability level (low, moderate and high) that is helpful when making clinical decisions regarding rehabilitation intervention ${ }^{7}$.

A global decrease in shoulder range of motion referring to the actual adherence of the shoulder capsule to the humeral head. Secondary causes include alteration of the supporting structures of and around the shoulder, autoimmune, endocrine or other systemic diseases ${ }^{8}$. Adhesive capsulitis is more common in diabetes mellitus ${ }^{2,9,10}$, and may be triggered by a rotator cuff tear, local trauma, myocardial infarction or hemiplegia, immobilization from any cause $\mathrm{e}^{2,11}$. It occasionally appears after recovery from neurosurgery ${ }^{4}$.

The histological features are reminiscent of Dupuytren's disease, with active fibroblastic proliferation in the rotator interval, anterior capsule and coraco- humeral ligament ${ }^{4}$. Three stages, each lasting 4-6 months, mark the clinical course. The progression of the disease is self- limiting and may occasionally resolve in partial restitution ${ }^{12}$.

The loss of range is multi planar, with external rotation and abduction being the most affected. Restricted passive external rotation as diagnostic of CFS would standardize and may clarify the clinical aspect of diagnosis ${ }^{13}$. Clinically the diagnosis should be suspected with progressive loss of range and diffuse pain despite conservative treatment measures9. Apart from slight wasting, the shoulder looks quite normal, tenderness is seldom marked. The shoulder is tender on palpation and both active and passive movements in all directions are restricted ${ }^{9}$. One clinical test alone is generally not sufficient to make a pathoanatomical diagnosis ${ }^{14}$. 
Idiopathic adhesive capsulitis is a common medical diagnosis for patients seeking physical therapy. Modalities used to treat adhesive capsulitis were dichotomized by pain predominant and stiffness-predominant classifications, which may be more useful than existing classifications ${ }^{13}$. Several treatment options are commonly used, but few have high level evidence to support them ${ }^{15}$. Physical therapy play an important role in the treatment of many shoulder pain including adhesive capsulitis $^{16}$. Clinical studies do not show clear effects of specific treatment. This may be caused by methodological weaknesses of the trials evaluated and that patient characteristics and placebo effects contribute to the diagnosis ${ }^{14}$. The transmission of drugs through the intact skin using ultrasound is called phonophoresis. Griffin and coworkers recommended phonophoresis treatment in common musculoskeletal diseases including adhesive capsulitis of shoulder ${ }^{17}$.

Phonophoresis is a variant of ultrasound in which biologically active substances are combined with the coupling medium in the hope that ultrasound will force the active material into tissue $^{18}$. For instance, NSAIDs gel is used in combination with coupling medium to enhance pain relief for local action. Although this technique has been in use since the 1960s, neither its effectiveness, penetration, optimal frequency, appropriate coupling mediums/ active materials, nor amount of material lost to the subcutaneous circulation is well established. Although some clinical studies report phonophoresis with a variety of agents successful in terms of improved shoulder range of motion and pain following treatment ${ }^{18}$. Perhaps mechanism of transdermal migration could involve increased cell permeability from the thermal effects of ultrasound. Ultrasonic coupling gel is mixed with various chemical substances to produce the phonophoresis coupling agent. Typical phonophoresis treatment parameters are similar to those of standard ultrasound: pulsed mode, 1 $\mathrm{MHz}$ transducer frequency, stroking technique, at $1-1.5 \mathrm{~W} /$ $\mathrm{cm}^{2}$, for approximately 5 to $10 \mathrm{~min}$ per site ${ }^{19}$.

Aetiology

The primary form of frozen shoulder having an unknown etiology and increased occurrence in patients with metabolic disorders and the secondary one being seen with poor injury or operation ${ }^{1,12,20}$.

\section{Risk factors}

- Prolonged immobilization ${ }^{1,3}$

- $\quad$ Middle age women (40-60 years $)^{1}$

- Diabetic patients $\mathbf{s}^{3,2,9}$

- Thyroid disease (Hyperthyroidism, Hypothyroidism $)^{1,3}$

- Trauma $^{2}$

- Stroke $^{3}$

- Cardiovascular disease ${ }^{1}$

- Myocardial infarction ${ }^{1,2}$

- Cervical disc disease ${ }^{21}$

- Intrathoracic disorders as TB, carcinoma, emphysema etc ${ }^{9,10}$

- Parkinsonism $^{1}$

- Post surgical condition due to immobilization ${ }^{1}$

\section{PATHOLOGY}

The pathophysiology involves a diffuse inflammatory synovitis with subsequent adherence of the capsule and a loss of the normal axillary pouch and joint volume, which leads to a significant loss of motion. Capsular contracture is thought to result from adhesion of the capsular surfaces or fibroblastic proliferation in response to cytokine production ${ }^{1}$.

In adhesive capsulitis, the capsule of the shoulder is thickened, and a mild chronic inflammatory infiltrate and fibrosis may be present ${ }^{9}$.

Modern work, such as that of Bunker, shows that adhesive capsulitis is primarily a fibrosing condition affecting the capsule (fibrous bag around a joint) of the shoulder joint. This leads to a tightening up of the coracohumeral ligament (one of the ligaments attaching the shoulder blade to the arm bone), which then restricts passive movement of the shoulder, especially the external rotation ${ }^{22}$.

\section{History taking and Physical examination}

Important points in the history include age, hand dominance, occupational and sport activities including heavy lifting or overhead repetitive movements, history of trauma, onset, location, character, duration, and radiation of the shoulder pain, aggravating and relieving factors, presence of night pain, and the effect on shoulder function. Associated symptoms, such as shoulder swelling, stiffness, restriction of movement, grinding, clicking, instability, or weakness, may also provide useful diagnostic clues ${ }^{3}$.

Physical examination of the shoulder should be thorough and as well as all areas of musculoskeletal system to exclude other causes of arthritis, it should include an assessment of these areas of the body where a source of referred pain suspected such as the upper abdomen in the case of shoulder tip pain. A systematic approach to the examination is important in order to fully assess all the structures around the shoulder itself. Both shoulders should be examined and any difference between the two sides in terms of power, stability and range of motion noted, taking into account the handedness of the patient. The peripheral pulse and neurological examinations including deep tendon reflexes, sensory and power in both upper limbs should be examined. Always screen the cervical spine in examining a case of shoulder pain ${ }^{23}$.

\section{INVESTIGATION}

A complete blood cell count, erythrocyte sedimentation rate, serum creatinine, serum uric acid and thyroid function tests are done as a screening panel. Further testing is done if the results suggest the possibility that the patient may have a systemic illness ${ }^{1}$. Adhesive capsulitis is more common among diabetics, random blood sugar or fasting and 2 hours after breakfast should be done to exclude diabetes mellitus ${ }^{9,10}$. If adhesive capsulitis is associated with other malignant condition, ESR is raised, otherwise ESR is normal ${ }^{24}$. RA test, c- reactive protein, urine and stool routine examination can be done to exclude Rheumatoid arthritis or seronegative spondyloarthropathy. 
Imaging of the shoulder

Plain films are useful as an initial screening test for patients with shoulder pain, ultrasound and MRI are suitable techniques for diagnosing rotator cuff disease $\mathrm{e}^{25}$.

$\mathrm{X}$ ray

Plain radiographs should include true anteroposterior, lateral view on abduction, axillary, and scapular Y views of the shoulder. The radiography reveals the width of the joint space, soft tissue swelling, periarticular erosions, soft tissue calcification, free joint bodies, periarticular swellings, degeneration changes, dislocation, osteoporosis ${ }^{1,26}$.

$\mathrm{X}$-rays are normal in adhesive capsulitis, unless they show reduced bone density from disuse. Their main value is to exclude other causes of a painful, stiff shoulder. The value of $\mathrm{X}$-rays in differential diagnosis was under-recognized ${ }^{13}$. The incidence of degenerative changes in the normal population makes it difficult to interpret diagnostic images ${ }^{14,27}$.

Ultrasonography :

Ultrasound provides a reliable and simple means of identifying rotator cuff pathology, impingement syndrome, shoulder instability, degenerative and inflammatory arthropathies, ${ }^{28}$ calcific tendinitis and biceps problems ${ }^{4}$. Non traumatic shoulder pain is usually diagnosed adequately from the medical history and a combination of clinical tests. When diagnostic imaging is required, ultrasound should be the first choice ${ }^{14}$.

\section{T Scan}

Computed Tomography (CT scan) is useful particularly when enhanced with intraarticular contrast, CT scan can identify cuff tears and labral detachments ${ }^{4}$.

\section{MRI}

MRI is the technique of choice for most other complex shoulder problems and for patients with normal plain films and nonspecific pain. Monoarthropathies, tumours and osteonecrosis will all be detected by $\mathrm{MRI}^{25}$. For patients with symptoms and signs suggesting instability, it can demonstrate associated anomalies of the capsule, labrum, glenoid and humeral head ${ }^{4}$. MRI is mainly indicated as part of a preoperative assessment and upon a specific indication. For diagnosis of the rotator cuff, MRI is as good as ultrasound ${ }^{14,27}$.

\section{Arthroscopy}

Arthroscopy can be useful to diagnose (and treat) intraarticular lesions, detachment of the labrum or capsule and impingement or tears of the rotator cuff. Arthroscopy is said to be the best means by which superior labrum, anterior and posterior (SLAP) tears may be diagnosed ${ }^{4}$. Arthroscopy is not useful for either diagnosis or treatment of adhesive capsulitis but may be useful for recognition of the stages of the disease ${ }^{29}$.

\section{Magnetic resonance arthrography}

Using magnetic resonance arthrography, a sensitivity of $91 \%$ and a specificity of $93 \%$ have been reported in the detection of pathological labral conditions. For identifying rotator cuff partial undersurface tears, MRA has been shown to be more sensitive and specific than MRI alone ${ }^{4}$. MRA is usually reserved for the assessment of shoulder instability ${ }^{25}$.
Differential Diagnosis: ${ }^{3,4,8,30,31,32,33}$

1. Cervical radiculopathy

2. Impingement syndrome

3. Calcifying supraspinatus tendinitis

4. Osteoarthritis of the acromioclavicular joint

5. Osteoarthritis of the glenohumeral joint

6. Rotator cuff tears/ arthropathy

7. Rheumatoid arthritis

8. Reactive arthritis

9. Recurrent dislocation of the shoulder

10. Infection in and around the shoulder

11. Tuberculosis of the shoulder joint

12. Biceps tendon instability and tendinitis

13. Neuralgic amyotrophy

14. Reflex sympathetic dystrophy syndrome

15. Milwaukee shoulder

16. Cardiac ischaemia

17. Chronic lung disease, Lung cancer

18. Post mastectomy

Treatment

Aim of treatment is directed to the shoulder for the purposes of:

- Relieving pain ${ }^{4}$

- Prevent further stiffening and restoring range of joint motion of shoulder ${ }^{4}$

- Restoring muscular strength and function of shoulder ${ }^{34}$

Individualized treatment is necessary following thorough evaluation of patient symptoms and stage of the disease ${ }^{35}$. As the condition is often self-limited, observation and reassurance may be considered; however, this may not be acceptable to many patients because of the painful and debilitating nature of the condition. Nonsurgical treatments include analgesics (eg, acetaminophen, nonsteroidal anti-inflammatory drugs), oral prednisolone, and intra-articular corticosteroid injections. Home exercise regimens and physical therapy are often prescribed. Arthroscopic capsular release has gained popularity over the years and offers a predictably good treatment in patients with adhesive capsulitis ${ }^{36}$. Surgical treatments include manipulation of the joint under anaesthesia and capsular release $\mathrm{e}^{7,12,15}$

Treatment should be adjusted to these stages. Recommendations include analgesics and joint injections in the first stage and physiotherapy in combination with manual therapy in the second and third stages ${ }^{12}$. Continuous passive motion (CPM) is an established method of preventing joint stiffness and of overcoming $i t^{37}$. The addition of deep heating to stretching exercises produced a greater improvement in pain relief, and resulted in better performance in the activities of daily living and in range of motion than superficial heating ${ }^{38}$. A rehabilitation model based on evidence and intervention strategies matched with irritability levels is proposed. Exercise and manual techniques are progressed as the patient's irritability reduces. Response to treatment is based on significant pain relief, improved satisfaction, and return of functional motion. Patients who do not respond or worsen should be referred for an intra-articular corticosteroid injection or passive exercise under interscalene block. Patients who have recalcitrant symptoms and disabling pain may respond to either standard or translational manipulation under anesthesia or arthroscopic release $\mathrm{e}^{7,12}$. 
Pharmacologic treatment: ${ }^{1,3,8,39}$

Nonsteroidal anti-inflammatory drugs (NSAIDs) for instance, Naproxen, piroxicam, indomethacin, diclofenac, ibuprofen etc. help to relieve pain and inflammation. Muscle relaxants are helpful in the early stages of the disease when spasm is predominant. Low-dose antidepressant medications (e.g., 10 $\mathrm{mg}$ of amitriptyline taken at night) may help to avoid a cycle of sleep disturbance leading to a chronic pain syndrome and fibromyalgia. Intra-articular steroid injection has proven benefit in the management of adhesive capsulitis. The early use of intraarticular corticosteroid injections during stage 1 and 2 in order to decrease the initial inflammatory stage in an attempt to reduce the development of fibrosis.

Exercise: ${ }^{4,40,41}$

The natural history of adhesive capsulitis is a matter of controversy. Management of true capsular restriction of motion (ie, true adhesive capsulitis) begins with gentle, progressive stretching exercises.

- Shoulder mobilizing exercises to increase range of motion are the following

a. Codman/ pendulum exercise

b. Wall climbing, pulley, wand exercises

c. Exercise in Chandler position

- Strengthening exercises when necessary in the form of resistant exercise with dumb-bell, weight, shoulder wheel etc.

Within the regained range of motion strengthening exercises are started as an isometric program progressing to resistant strengthening through the full arc of motion.

\section{- Codman/ pendulum exercise}

These are self mobilization techniques that use the effects of gravity to distract the humerus from the glenoid fossa. They help relieve pain through gentle traction and oscillating movements and provide early motion of joint structures and synovial fluid. No weight is used initially. When the patient tolerates stretching, a weight is added to the hand or as wrist cuffs to cause further distraction force. To direct the stretch force to glenohumeral joint, stabilize the scapula against the thorax manually or with a belt.

\section{- Wall climbing exercise}

Walking fingers up the wall while the shoulder is held in abduction and in forward flexion. Line or length is marked in the $1^{\text {st }}$ visit which is monitored thereafter in subsequent follow up visits.

\section{- Shoulder wheel}

Circumduction of the shoulder joint clockwise and anticlockwise using shoulder wheel. Also resistance can be applied within the wheel and exercise can be progressively done against resistance to strengthening the muscles as well.

\section{- Wand exercises}

When a patient has voluntary muscle control in an involved upper extremity but needs guidance or motivation to complete the ranges of motion in the shoulder, a dowel rod (cane, wooden stick, T- bar, or similar object) can be used to provide assistance.

\section{- Overhead pulley exercise}

Pulley system can be effectively used to assist an involved extremity in performing ROM. Stretching the shoulder flexion, abduction, internal and external rotation by using overhead pulley.

Three to four types of exercises may be advised at a time for few repetitions each type three or four times daily, otherwise exercise itself would increase pain if it is not advised and carried out properly.
Physical measures: ${ }^{1}$

- Deep heat such as ultrasound, microwave diathermy, short wave diathermy

- Superficial heat such as infrared, hot pack and wax bath

- Ice pack

- Transcutaneous electrical nerve stimulation

Thermotherapy in adhesive capsulitis

The physiological responses of the heat are as follows: ${ }^{17,19}$

1. Increases blood flow by vasodilatation, so increases oxygen and nutrition at the site

2. Decreases chronic inflammation by washing out the inflammatory exudates

3. Decreases pain and joint stiffness

4. Increases tendon and collagen extensibility

Ultrasound therapy: ${ }^{17,19}$

Ultrasound is a deep heating modality that uses high frequency acoustic vibration above the human audible spectrum (i.e. $>20000 \mathrm{~Hz})^{19}$. Therapeutic ultrasound involves the use of high frequency acoustic energy to produce thermal and non thermal effects in tissue. The most commonly used frequency is $1 \mathrm{MHz}^{17,19}$. A transducer operating at therapeutic frequencies will produce a beam with a greater angle of divergence if the diameter of the transducer is small than if it is large ${ }^{17}$. The World Health Organization and the International Electrical Commission both recommend limiting spatial average intensity to $3 \mathrm{~W} / \mathrm{cm}^{2}{ }^{2}{ }^{19}$ Most clinically used intensities of therapeutic ultrasound are in the $0.1-2.5 / 3 \mathrm{~W} / \mathrm{cm}^{2}$ according to machine. The wave length is approximately 0.15 $\mathrm{cm}^{17}$. duration generally over 5 to 10 minutes per site to obtain optimal heating of joint tissues located right in the front of the bone. Temperature reaches up to $46^{0} \mathrm{C}\left(114.8^{0} \mathrm{~F}\right)$ in deep tissues are easily achieved with ultrasound ${ }^{19}$.

Phonophoresis: ${ }^{17,42}$

It is also known as sonophoresis or ultrasonophoresis. This implies the movement of drugs through intact skin into the subcutaneous tissues under the influence of ultrasound. Many drugs are absorbed through the skin very slowly, using usually high frequency ultrasound. Phonophoresis relies on perturbation of the tissues causing more rapid particle movement through the skin, thus encouraging absorption of the drug. Topical NSAIDs like naproxen/ diclofenac gel can be introduced through the skin for relief of pain locally specially in patients who have peptic ulcer disease, bronchial asthma or renal failure. UST itself is relieving pain and stiffness by its mechanism of action, but here, with addition of NSAIDs gel, it gives an additional effect of pain relief of that area. Drugs used are steroids, anti-inflammatories and local anaesthetics ${ }^{42}$.

\section{PROGNOSIS}

Adhesive capsulitis is regarded as distinct clinical entity showing a benign and regular course. ${ }^{31}$ Patients with more severe pain and functional limitations before treatment had relatively worse outcomes ${ }^{43}$. It is a self limiting disease, may take up to 18 months to improve completely ${ }^{4}$.

\section{PREVENTION}

To prevent the problem, a common recommendation is to keep the shoulder joint fully moving. Often a shoulder will hurt when it begins to freeze. Because pain discourages movement, further development of adhesions that restrict movement will occur unless the joint continues to move full range in all directions (adduction, abduction, flexion, rotation and extension). Physical therapy in the form of exercises with few repetitions two to three times daily could prevent the development of adhesive capsulitis.

\section{DISCLOSURE}

All the authors declared no competing interest. 


\section{REFERENCES}

1. Martin SD, ThornhillnTS. Shoulder pain. In: Firestein GS, Budd RC, Harris ED etal. Kelley’s Textbook of Rheumatology. $8^{\text {th }}$ ed. Elsevier: Saunders; 2009; 607-8.

2. Doherty M, Ralston SH. Musculoskeletal disease. In: Colledge NR, Walker BR, Ralston SH (editors). Davidson's principles \& practice of Medicine. $21^{\text {st }}$ edition. Elsevier: Churchill Livingstone; 2010;1069.

3. Fam AG. The Shoulder. In: Fam AG, Lawry GV, Kreder HJ. Musculoskeletal examination and joint injection Techniques. $1^{\text {st }}$ ed. Elsevier: Mosby; 2006; 21-2.

4. Cole A, Pavlou P. The shoulder and pectoral girdle.In.Solomon L, Warwick DJ, Nayagam S. Apley's System of Orthopaedics and Fractures. $9^{\text {th }}$ ed. Hodder Arnold; 2010; 351-353.

5. Arslan S, Celiker R. Comparison of the efficacy of local corticosteroid injection and physical therapy for the treatment of adhesive capsulitis. Rheumatol Int. 2001; 21(1): 20-3.

6. Zuckerman JD, Rokito A. Frozen shoulder: A consensus definition. J Shoulder Elbow Surg. 2010 Nov 2

7. Kelly MJ, McClure PW, Leggin BG. Frozen shoulder: evidence and a proposed model guiding rehabilitation. J Orthop Sports Phys Ther. 2009; 39 (2): 135- 48

8. Siegel LB, Cohen NJ, Gall EP. Adhesive Capsulitis: A Sticky Issue. Am Fam Physician. 1999; 59 (7): 1843-1850.

9. Langford CA, Gilliland BC. Periarticular Disorders of the Extremities. In: Fauci AS, Longo DL, Kasper DL, et al (editors). Harrison's Principles of Internal Medicine. $18^{\text {th }}$ ed. Mc Graw Hill; 2012; 2861-2862.

10. Tighe CB, Oakley WS. The prevalence of a diabetic condition and adhesive capsulitis of the shoulder. Southern Medical Journal. 2008; 101 (6): 591- 595.

11. YAMSHON LJ. Frozen shoulder: methods for bringing about early mobilization. Calif Med. 1958; 89 (5): 333- 4.

12. Schultheis A, Reichwein F, Nebelung W. Frozenshoulder. Diagnosis and therapy. Orthopade. 2008; 37 (11): 1065- 72.

13. Hanchard NC, Goodchild L, Thompson J, O'Brien T, Davison D, Richardson C. A questionnaire survey of UK physiotherapists on the diagnosis and management of contracted (frozen) shoulder. Physiotherapy. 2011; 97 (2): 115- 25.

14. Brox JI, Sunde P, Schroder CP, Engebresten K, Skare O, Ekeberg OM, etal. Non-traumatic shoulder pain. Tidsskr Nor Laegeforen. 2010; 130 (21): 2132- 2135.

15. Ewald A. Adhesive capsulitis: a review. Am Fam Physician. 2011;83 (4): 417-22.

16. Strang MH. Physiotherapy of the shoulder complex. Baillieres Clin Rheumatol. 1989; 3 (3):669-80.

17. Justus F, Lehmann and Barbara J, Lateur D. The Clinical Use of Heat and Cold in various Conditions. In: kottke FJ, Lehmann JF (editors). KRUSEN'S HANDBOOK OF PHYSICAL MEDICINE and REHABILITATION. $4^{\text {th }}$ ed. Philadelphia: W.B.SAUNDERS; 1990; 285- 352.

18. Basford JR. Therapeutic Physical Agents. In: Delisa JA, Gans BM (editors). Physical Medicine \& Rehabilitation principles and practice. $4^{\text {th }}$ ed. Lippincott: Williams \& Wilkins; 2005; 257.

19. David C, Weber and Kurtis M, Hoppe. Physical Agent Modalities. In: Braddom RL (editor). Physical Medicine \& Rehabilitation. $3^{\text {rd }}$ ed. Elsevier: Saunders; 2007; 464- 472.

20. Huygen F, Patijn J, Rohof O, Lataster A, Mekhail N, Vankleef M, et al. Painful shoulder complaints. Pain Pract. 2010; 10 (4): 318- 26.

21. Guler-Uysal F, Kozanoglu E. Comparison of the early response to two methods of rehabilitation in adhesive capsulitis. SWISS MED WKLY. 2004; 134: 353-358.

22. http://www.thephysiotherapysite.co.uk/physiotherapy/shoulders/pathology-of-frozen-shoulder (retrieved on 6/12/2011)

23. McRae R. Clinical Orthopaedic Examination. $6^{\text {th }}$ ed. Elsevier: Churchill Livingstone; 2010; $52-57$.

24. http://www.refhelp.scot.nhs.uk/index.php?option=com_content\&task=view\&id=431\&Itemid $=237($ retrieved on 18/12/2011)

25. Ostlere S. Imaging the shoulder. Imaging. 2007; 19: 191-200.

26. Armstrong P, Wastie ML. Diagnostic Imaging. $4^{\text {th }}$ ed. Blackwell Science; 1998; 333-50.

27. Grubbs N. Frozen shoulder syndrome: a review of literature. J Orthop Sports Phys Ther. 1993; 18 (3): 479- 87.

28. Daenen B, Houben G, Bauduin E, Bauduin E, Lu KV, Meulemans JL. Ultrasound of the shoulder. JBR-BTR. 2007; 90: 325-337.

29. Neviaser RJ, Neviaser TJ. The frozen shoulder. Diagnosis and management. Clin Orthop Relat Res. 1987; 223: 59- 64.

30. http://expertscolumn.com/content/frozen-shoulder-symptoms-and-differential-diagnosis (retrieved on 9/12/2011)

31. Green S, Buchbinder R, Hetrick S. Physiotherapy interventions for shoulder pain. Cochrane Database Syst Rev. 2003; (2): CD004258.

32. Peterson A, Kincaid JC. Rehabilitation of patients with neuropathies. In: Braddom RL (editor). Physical Medicine \& Rehabilitation. $3^{\text {rd }}$ ed. Elsevier: Saunders; 2007; 1086- 87.

33. Genta M S, Gabay C. Milwaukee shoulder. N Engl J Med. 2006; 354.

34. Kibler WB. Shoulder rehabilitation: principles and practice. Med Sci Sports Exerc. 1998; 30 (4 Suppl): S40-50.

35. Sheridan MA, Hannafin JA. Upper extremity: emphasis on frozen shoulder. Orthop Clin North Am. 2006; 37 (4): 531-9.

36. Tasto JP, Elias DW. Adhesive capsulitis. Sports Med Arthrosc. 2007; 15 (4): 216- 21.

37. Dundar U, Toktas H, Cakir T, Evcik D, Kavuncu V. Continuous passive motion provides good pain control in patients with adhesive capsulitis. Int J Rehabil Res. 2009; 32 (3): 193-198.

38. Leung MS, Cheing GL. Effects of deep and superficial heating in the management of frozen shoulder. J Rehabil Med. $2008 ; 40$ (2): $145-50$.

39. Finnoff. Musculoskeletal problems of the upper limb. In: Braddom RL (editor). Physical Medicine \& Rehabilitation. $3^{\text {rd }}$ ed. Elsevier: Saunders; 2007; 834- 835.

40. Kisner C, Colby LA. Therapeutic exercise foundations and techniques. $4^{\text {th }}$ ed. Jaypee; $43-49,282$.

41. Hollis M, Kitchen SS. Biomechanics. In: Hollis M, Flecher-Cook P (editor). Practical Exercise Therapy. $4^{\text {th }}$ ed. Blackwell Science; 1999; $29-31$.

42. Robertson V, Ward A, Low J, Reed A. Electrotherapy Explained Principles and Practice. $4^{\text {th }}$ ed. Elsevier: Butterworth Heinemann; 2009; 251-297.

43. Griggs SM, Ahn A, Green A. Idiopathic adhesive capsulitis. A prospective functional outcome study of nonoperative treatment. J Bone Joint Surg Am. 2000; 82-A (10): 1398-407. 